\title{
Radiative signatures from impact of Comet Shoemaker-Levy-9 on Jupiter
}

\author{
Thomas J. Ahrens, Toshiko Takata, John D. O'Keefe \\ Lindhurst Laboratory of Experimental Geophysics, Seismological Laboratory, California Institute of Technology, Pasadena, \\ California 91125
}

Glenn S. Orton

Jet Propulsion Laboratory, California Institute of Technology, Pasadena, California 91109

\begin{abstract}
The visible optical power emitted from the expansion plumes from 0.4 and $2 \mathrm{~km}$ diameter fragments of ShoemakerLevy are expected to be, $\sim 25 \%$ and comparable to, the visible solar flux reflected from Jupiter, respectively, for several minutes, and could be easily observed by sensors on the Galileo spacecraft. Earth-based observers can detect these plumes as these expand over the SW limb of Jupiter and come into earth view some minutes after impact!
\end{abstract}

\section{Introduction}

This is the first time in history an astronomical object, CometShoemaker-Levy 9 (SL-9), has been discovered in the sky [Shoemaker et al., 1993] which is predicted to impact a planet, Jupiter, at a pre-determined time and position. SL-9 upon close passage, within the Roche limit, of Jupiter on 8 July 1992 brokeup into some 20 fragments. These are now completing a 2 year orbit of Jupiter and SL-9 fragments will impact (at $60 \mathrm{~km} / \mathrm{sec}$ ) Jupiter for some six days starting July 16, 1994 [Yeomans and Chodos, 1993]. (The impact velocity is only slightly in excess of the escape velocity because the SL- 9 encounter velocity at large distances from Jupiter is small compared to Jupiter's $60 \mathrm{~km} / \mathrm{sec}$ escape velocity). As viewed from the Earth, SL-9 fragments impact the "back side" of Jupiter. However, the impact points will rotate over Jupiter's limb and come into view of the Earth 7 to 13 minutes later.

Previously, Ahrens et al. [1994] and Takata et al. [1994], modeled the interaction of the SL-9 fragments with Jupiter's atmosphere (Fig. 1) in three stages: 1) the passage and transfer of the kinetic energy of the comet fragment to the atmosphere, 2) the expansion and subsequent rise of the atmosphere heated by the stopping of the comet, and 3 ) the radiative signal which is emitted by the heated plume.

We used the Smoothed Particle Hydrodynamics (SPH) method to calculate the passage and stopping of 2- and 10-km diameter (1 $\mathrm{Mg} / \mathrm{m}^{3}$ density) ice comet fragments impacting Jupiter at an angle of $40^{\circ}$ from the zenith at $60 \mathrm{~km} / \mathrm{s}$. In the present paper we scale these "entry" results as described below to a $0.4 \mathrm{~km}$ diameter fragment and then performed plume and radiative calculations on the effects of this diameter object impacting Jupiter. Taking into account recent trajectory refinements we predict the radiative signal from SL-9 fragment impacts and their observabilities from the Galileo spacecraft and Earth.

\section{Copyright 1994 by the American Geophysical Union.}

Paper number 94GL01578

0094-8534/94/94GL-01578\$03.00

\section{Approach}

Because of the contrast in length scales of the $0.4 \mathrm{~km}$ diameter comet and the $\sim 10^{2} \mathrm{~km}$ scale of the atmosphere with which it interacts, we were unable to conduct the stage 1 (entry) calculation for this case.

To describe the plume and radiation from a $0.4 \mathrm{~km}$ cometary fragment we fit ablation theory [Bronshten, 1983] to our previous SPH calculations [Ahrens et al., 1994; Takata et al., 1994]. The loss of bolide mass, $m$, with time, $t$, is described by

$$
\mathrm{dm} / \mathrm{dt}=-\sigma C_{\mathrm{D}} S \rho_{\mathrm{a}} \mathrm{V}^{3} / 2
$$

where $C_{D}$ is the drag coefficient, $S$, the bolide cross-sectional area, $\rho_{\mathrm{a}}$ is the local atmospheric density, $\mathrm{V}$ is the bolide velocity, and $\sigma$ is the ablation parameter which is given by

$$
\sigma=\alpha /\left(C_{D} \xi\right)
$$

where $\alpha$ is the fraction of the comets' kinetic energy taken up by ablation, and $\xi$ is the specific energy of ablation [Bronshten, 1983]. The ablation parameter, $\sigma$, here is used to merely parameterize a complex series of meteor processes only some of which are code models. We parameterize from our SPH calculations such that $\sigma \cong 10^{-9} \mathrm{~s}^{2} / \mathrm{m}^{2}$. Peak penetration pressure (P, bars) levels within Jupiter's atmosphere are calculated to be 30,200 and 800 bars for $D=0.4,2$, and $10 \mathrm{~km}$ (diameter cometary fragments). Our fits to the SPH penetration results yield

$$
\log _{10} \mathrm{P}(\text { bars }) \cong 2+0.9 \log _{10} \mathrm{D}(\mathrm{km})
$$

Thus penetration to below Jupiter's cloud depth occurs for a 0.4 $\mathrm{km}$ comet fragment. Moreover, we estimate that a $0.1 \mathrm{~km}$ diameter fragment will just penetrate to below the clouds. These results approximately agree within factors of $\sim 5$ and $\sim 2$ in ambient Jupiter pressure (greater penetration) with those of MacLow and Zahnle [1994] and Crawford et al [1994] but give less penetration to ambient pressure levels by a factor of 2 than Vickery [1993]. However, the present results and the two-dimensional calculations of MacLow and Zahnle, Crawford et al., and Vickery, yield penetration to Jupiter pressures some $10^{3}$ greater than calculated by Sekanina [1993]. Basically, Sekanina assumes comet nucleii are lower density, have larger ablation parameters and hence are stopped at higher altitudes in Jupiter's atmosphere.

\section{Expansion Driven Plume}

To describe the driven upward expansion flow fields (plumes) which result from the energy deposition, we constructed a second 

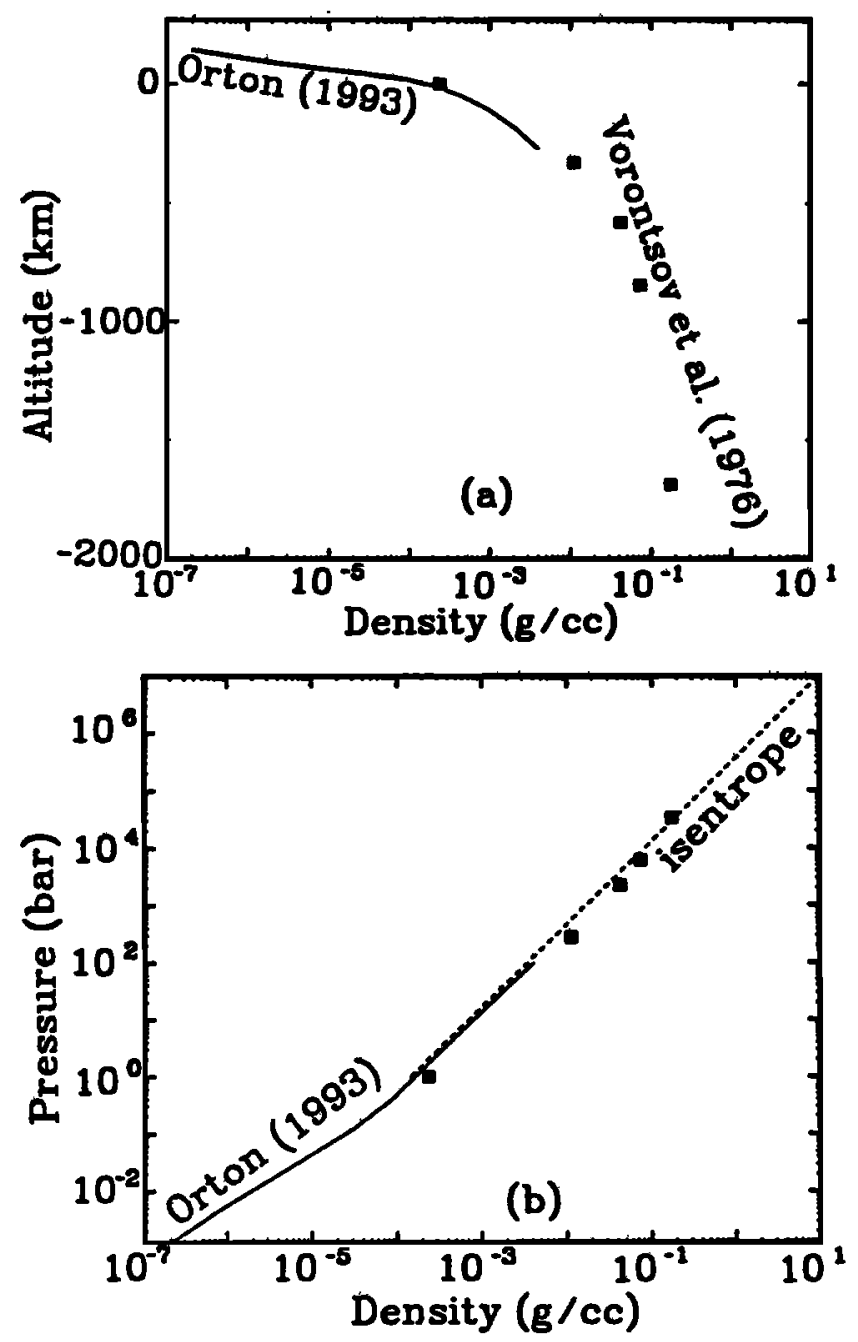

Fig. 1. Models of Jupiter atmosphere (a) density versus altitude with respect to zero altitude at 1 bar. (b) density versus pressure, the square symbols in (b) come from Vorontsov et al. [1976] The solid curve is being used as a working atmospheric model of Jupiter in the Galileo project.

SPH grid of particles and assigned excess (relative to ambient) energies to particles in a linear array lying at $40^{\circ}$ to zenith (details are given in Takata et al. [1994]). Although impactor dimensions for the 0.4 and $2 \mathrm{~km}$ fragments differ by a factor of 5 , the plume dimensions from a 0.4 and $2.0 \mathrm{~km}$ impactor do not differ by such a large factor. For example, at 90 secs (after the impactor fragments have come to rest) the $0.4 \mathrm{~km}$ object induces a plume which extends to $250 \mathrm{~km}$ height $(0 \mathrm{~km}=1$ bar) and has a maximum width of $200 \mathrm{~km}$. In contrast, at a similar time, the plume from the $2 \mathrm{~km}$ diameter fragment has an altitude of $350 \mathrm{~km}$ and a maximum width of $500 \mathrm{~km}$.

The upward gas ejecta velocities (at some 90 seconds after the impactor fragments have come to rest) are, however, a factor of 5 different. These are 2 and $10 \mathrm{~km} / \mathrm{sec}$ for impact of 0.4 and $2 \mathrm{~km}$ diameter cometary fragments, and hence, neither cometary vapor or Jovian atmosphere will escape the planet.

\section{Radiative Signatures}

Starting with plume calculations of 0.4- and 2-km SL-9 fragments , we have considered each of the $-10^{5}$, SPH particles, as greybody radiator discs and calculated the total normal radiative power as a function of wavelength (e.g. Fig. 2). Since we are not accounting for the absorption of $\mathrm{CH}_{4}$ or Rayleigh scattering from $\mathrm{H}_{2}$, the present calculations are an upper bound to the actual radiating power. However, most of the hot materials in the plume, are at, or above, the elevations such that there will be minimal absorption effects of clouds. In the case of the $2 \mathrm{~km}$ comet fragment impact plume, its particle velocity will allow it to achieve an altitude of $3000 \mathrm{~km}$ within several minutes. In contrast, a $0.4 \mathrm{~km}$ diameter impactor produces a plume with an upward velocity of $-2 \mathrm{~km} / \mathrm{sec}$ and thus will rise only to $-300 \mathrm{~km}$ height.

At 0.4 microns, the peak radiated power, $P$, for $0.4,2$, and 10 $\mathrm{km}$ diameter ( $1 \mathrm{Mg} / \mathrm{m}^{3}$ density, ice) cometary fragments are related to their impact energy $\mathrm{E}$ (ergs) by:

$\log _{10} P\left(\mathrm{ergs} / \mathrm{sec} / \mathrm{cm}^{3}\right)=144.4-10.74 \log _{10} E+0.198\left(\log _{10} E\right)^{2}$.

This fit has a comrelation parameter, $\mathbf{R}$, of 0.996 .

\section{Radiative Predictions}

In July 1994, the Galileo spacecraft will be in a good position (1.6 AU from Jupiter) to observe the plumes from SL-9 fragment collisions. The flux emitted by the plumes from 0.4 and $2-\mathrm{km}$ diameter fragments are $-25 \%$ and, approximately equal to, the solar visible ( 0.4 microns) flux reflected by Jupiter at $90 \mathrm{sec}$ after the impactor fragments come to rest. Because of over-expansion of the plume, the SPH particles stop interacting in our model calculation (at $90 \mathrm{~s}$ ) and are in essentially ballistic motion (as in Knudsen flow). Hence, our present method does not describe the time history of the radiative signals. At 3 microns the power from the 0.4 and $2 \mathrm{~km}$ impactor plumes are comparable to, and $10^{2}$, times Jupiter's reflected power, respectively. At 10 microns, the radiant power from the 0.4 and $2 \mathrm{~km}$ impactor plumes are 0.1 and -2 times Jupiter's thermal emission power. Thus, observations by Galileo instruments are technically feasible for a large number of the fragments if they are as dense as we have assumed.

Recent refinements of the orbit of SL-9 (D. Yeomans, private communication) indicate direct earth-based observations of the impact-induced plumes from 2 and $0.4 \mathrm{~km}$ comet fragments are possible some 3 minutes after impact as these rise to 1800 and 360 km above the planet. At this time, terrestrial observers can see the

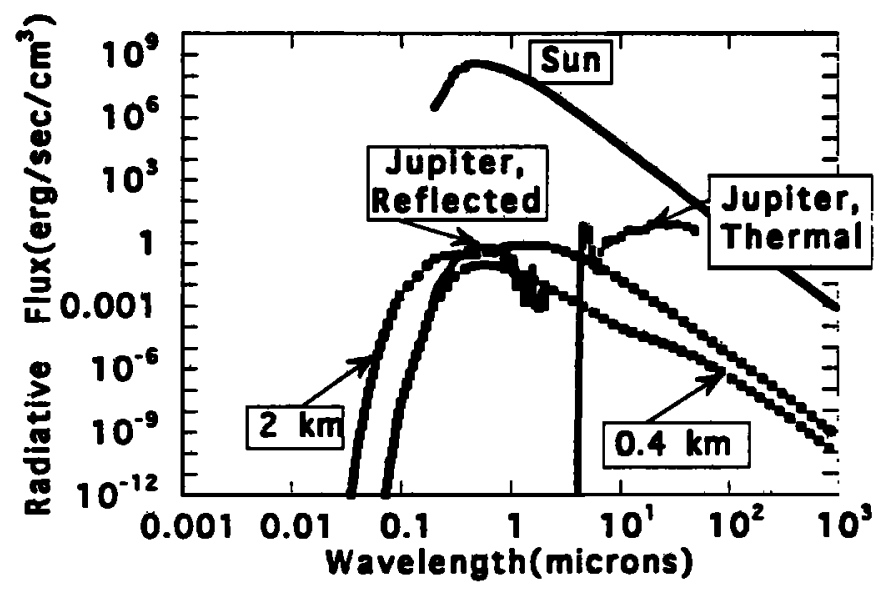

Fig. 2. Radiative power output of ejecta plume from 0.4 and $2 \mathrm{~km}$ diameter cometary fragments at $90 \mathrm{sec}$ after the impactor fragments come to rest. Radiative power output of solar and Jupiter discs is indicated. In the case of Jupiter, we indicate separately the reflected radiation and thermal radiation. 
plume from an object that impacted $6^{\circ}$ past the limb, if it is 120 $\mathrm{km}$ or more above the 1-bar pressure level horizon. Thus, it is likely, even given some uncertainty of the depth of impact penetration, that plumes from the 2 and $0.4 \mathrm{~km}$ fragments will be observable from the earth!

Three minutes after impact, the thermal radiation generated by the unresolved fireball from a 2-km impactor should be approximately $2-3 \mathrm{erg} \mathrm{sec}^{-1} \mathrm{~cm}^{-3}$ between 0.5 and 3 microns (e.g. a $\mathrm{V}$ magnitude of -1.9 to -2.4 ), falling off to $0.08 \mathrm{erg} \mathrm{sec}^{-1} \mathrm{~cm}^{-3}$ at 10 microns. At this same time, the thermal radiation generated by an unresolved plume from the $400-\mathrm{m}$ impactor is $\sim 0.1$ lower (e.g. $\mathrm{V}$ magnitudes of +0.1 to +0.6 ). For comparison, the radiation from the impact induced plumes after 3 minutes for $2-\mathrm{km}$ and $400-\mathrm{m}$ impactors are, thus $10^{2}$ and $10^{1}$ brighter than an average 2-arcsec square segment of Jupiter in the visible and near-infrared, respectively.

Finally, we assess the detectability of "indirect" radiation reflected from the satellites and consider Io in detail. Thus, for a 2-km object, the visible radiant power from the plume reflected from Io's surface is $-3 \times 10^{5} \mathrm{ergs} / \mathrm{sec} / \mathrm{cm}^{3}$, or some $0.1 \%$ of the amplitude of the incident sunlight. The near-infrared 2-km objectgenerated plume radiance is comparable to its amplitude in the visible, but the drop-off of the solar spectrum is sufficient to make the radiant power $\sim 1 \%$ of the incident solar radiation. A $0.4 \mathrm{~km}$ object will generate a plume whose near-infrared radiant power is $0.01 \%$ of the incident solar radiation. Thus, the earth-based reflected light from Jupiter's satellite measurements of the plume radiances make their observation possible, but technically difficult.

Acknowledgments. David Stevenson, John Spencer, Kevin Zahnle, Torrence Johnson, and two anonymous reviewers provided many helpful suggestions. We thank K. Zahnle and M. MacLow, and G. Field and A.
Ferrara for their preprints, and P. Weissman, M. A'Hearn, and K. Ziemelis for comments on the manuscript. Research supported by NASA and Cray Research Corp. Contribution number 5344, Division of Geological and Planetary Sciences.

\section{References}

Ahrens, T. J., T. Takata, J. D. O'Keefe, and G. S. Orton, Comet Shoemaker-Levy 9 impact on Jupiter, Geophys. Res. Lett., in press, 1994.

Bronshten, V. A., Physics of Meteoric Phenomena, 356 pp., D. Reidel Publishing Co., Boston, 1983.

Crawford, D. A., T. G. Trucano, M. B. Boslough, M. E. Kipp, and J. M. McGlaun, The influence of body shape on the deformation and breakup of Comet Shoemaker-Levy 9 fragments as they enter the Jovian atmosphere, Lunar \& Planet. Sci. (abstract), XXV, 295-296, 1994.

MacLow, M.-M., and K. Zahnle, The collision of Jupiter and Comet Shoemaker-Levy-9, Icarus, in press, 1994.

Sekanina, Z., Disintegration phenomena expected during collision of Comet Shoemaker-Levy 9 with Jupiter, Science, 262, 382-387, 1993.

Shoemaker, C. S., E. M. Shoemaker, and D. H. Levy, Comet ShoemakerLevy, LAU Circ., 5725, 1993.

Takata, T., J. D. O'Keefe, T. J. Ahrens, and G. S. Orton, Comet Shoemaker-Levy-9: Impact on Jupiter and plume evolution, Icarus, in press, 1994.

Vickery, A., Numerical simulation of a comet impact on Jupiter, EOS, 74, $391,1993$.

Vorontsov, S. V., V. N. Zharkov, and V. M. Lubimov, The freeoscillations of Jupiter and Saturn, Icarus, 27, 109-118, 1976.

Yeomans, D. K., and P. Chodos, Minor Planet Circ. No., 22197, 1993.

T. J. Ahrens, T. Takata, and J. D. OKeefe, Lindhurst Laboratory of Experimental Geophysics, Seismological 252-21, California Institute of Technology, Pasadena, CA 91125

G. S. Orton, Jet Propulsion Laboratory, Pasadena, CA 91109

(Received May 18, 1994; accepted June 2, 1994) 